\title{
Cognitive effects of electro-acupuncture and pregabalin in a trigeminal neuralgia rat model induced by cobra venom
}

This article was published in the following Dove Press journal:

Journal of Pain Research

8 August 2017

Number of times this article has been viewed

\author{
Ruo-Wen Chen ${ }^{1,2}$ \\ Hui Liu ${ }^{2}$ \\ Jian-Xiong $A n^{1,2}$ \\ Xiao-Yan Qian² \\ Yi-De Jiang ${ }^{2}$ \\ Doris K Cope ${ }^{3}$ \\ John P Williams ${ }^{3}$ \\ Rui Zhang' \\ Li-Na Sun' \\ 'Department of Anesthesiology, \\ Weifang Medical University, Weifang \\ City, Shandong, ${ }^{2}$ Department of \\ Anesthesiology, Pain Medicine and \\ Critical Care Medicine, Aviation \\ General Hospital of China Medical \\ University and Beijing Institute of \\ Translational Medicine, Chinese \\ Academy of Sciences, Beijing, China; \\ ${ }^{3}$ Department of Anesthesiology, \\ University of Pittsburgh School of \\ Medicine, Pittsburgh, PA, USA
}

Objective: The objective of this study was to investigate the effects of electro-acupuncture (EA) and pregabalin on cognition impairment induced by chronic trigeminal neuralgia (TN) in rats. Design: Controlled animal study.

Setting: Department of Anesthesiology, Pain Medicine and Critical Care Medicine, Aviation General Hospital of China Medical University.

Subjects: Forty adult male Sprague Dawley rats.

Methods: Rats were randomly divided into four groups. The TN model was induced by administration of cobra venom to the left infraorbital nerve. On postoperative day 14, either EA or pregabalin was administered, free behavioral activities were observed. Spatial learning and memory abilities were determined in the Morris water maze. The ultrastructural alterations of the Gasserian ganglion, medulla oblongata and hippocampus were examined by electron microscopy. The changes on long-term potentiation were investigated.

Results: After treatment, the exploratory behavior increased and the grooming behavior decreased $(P<0.05)$ for the EA group and pregabalin group compared with the cobra venom group; moreover, demyelination of neurons in Gasserian ganglion and medulla oblongata was reversed. The number of platform site crossings, the average percentages of time in the target quadrant and the field excitatory postsynaptic potential slopes increased $(P<0.05)$ in the EA group compared to the cobra venom group. However, the pregabalin group showed no differences compared to the cobra venom group $(P>0.05)$. Vacuolar degeneration in the hippocampal neurons was mild in the EA group, while it was severe in the pregabalin group.

Conclusion: EA and pregabalin could alleviate TN induced by cobra venom. EA could also inhibit the cognition deficit induced by TN, while pregabalin could not.

Keywords: TN, cognition dysfunction, EA, pregabalin, LTP

\section{Introduction}

Cognitive dysfunction is a common complication of chronic neuropathic pain, which involves changes in both nerve electrophysiology and anatomy of the brain. ${ }^{1-3}$ Our previous study revealed that chronic trigeminal neuralgia in rats induced by cobra venom impairs spatial learning and memory function as assessed by the Morris water maze test. ${ }^{1}$ However, since the underlying mechanism is unclear, there is a lack of effective therapeutic strategies to improve deficits in spatial learning and memory.

Electro-acupuncture (EA) is effective in the treatment of patients with chronic pain, such as low back pain, fibromyalgia and chronic pelvic pain. ${ }^{4-6}$ Previous work has shown that EA can remarkably improve cognition in rats in a model of Parkinson's disease. The mechanisms were possibly associated with protecting cholinergic neurons in the
Correspondence: Jian-Xiong An Department of Anesthesiology, Pain Medicine and Critical Care Medicine, Aviation General Hospital of China Medical University, and Beijing Institute of Translational Medicine, Chinese Academy of Sciences, Beiyuan Rd 3\#, Beijing 100012 , China

Tel +86 I 38 0I 28 I750

Fax +8610 59520393

Email anjianxiong@yahoo.com 
central nervous system and elevating the activity of choline acetyltransferase. ${ }^{7-9}$ Additionally, EA at the Baihui acupoint was observed to markedly ameliorate cognitive impairments, reduce the aberrant overexpression of $\beta$-amyloid and inhibit neuronal apoptosis in APP/PS1 transgenic mice with Alzheimer's disease. ${ }^{10}$ All these studies suggest that EA may be a cost-effective alternative in relieving neuropathic pain and improving cognitive function.

Pregabalin is a type of antiepileptic medication that is commonly used for pain management. Previous studies have indicated that pregabalin appears to be effective in the treatment of trigeminal neuralgia. ${ }^{11-13}$ However, there are various hypotheses regarding the treatment of persistent pain and the effects on cognitive function. Some animal studies have shown that pregabalin did not modify the cognitive process performance or influence short-term memory of mice in the Y-maze test. ${ }^{14}$ Yet, other investigators demonstrated that pregabalin induces mild negative cognitive effects in healthy volunteers. ${ }^{15}$

In this study, we used behavioral testing to confirm the analgesic effects of EA and pregabalin in a rat model of trigeminal neuralgia induced by cobra venom. The Morris water maze task was conducted to evaluate the spatial learning and memory ability of rats after either EA or pregabalin treatment. In addition, we discuss the possible mechanisms of EA and pregabalin in improving cognitive impairment induced by chronic trigeminal neuralgia in rats.

\section{Methods}

All experiments were approved by the Ethical Committee of Aviation General Hospital of China Medical University and were in accordance with the Guidelines for the Care and Treatment of Laboratory Animals of the US National Institutes of Health.

\section{Animals}

The experiments were performed on 40 male Sprague Dawley rats (weighing 200-260 g), which were obtained from the Laboratory Animal Center of the Academy of Military Medical Sciences. The Animal Care and Use Committee (Beijing, China) approved all animal protocols. The animals were housed together with freely available food and water before surgery for 1 week under controlled conditions (temperature $22^{\circ} \mathrm{C}-24^{\circ} \mathrm{C}$ and 7 am to $7 \mathrm{pm}$ alternating light-dark cycle). The rats were randomly divided into four groups: sham-operated $(n=10)$, cobra venom $(n=10)$, EA $(n=10)$ and pregabalin $(n=10)$.

\section{Trigeminal neuralgia model establishment}

The animal model was established according to the procedure reported by An et al. ${ }^{16}$ Forty rats were anesthetized by an intraperitoneal injection of chloral hydrate $(0.8 \mathrm{~mL} / 100 \mathrm{~g}$ body weight; Sigma-Aldrich, St Louis, MO, USA). The incision was made $\sim 1 \mathrm{~cm}$ in length radically along the superciliary arch superior to the skin margin, exposing the fossa orbitalis and nasal bone. The content of the orbit was then deflected with a glass rod. The infraorbital nerve was freely dissected at its rostral extent in the orbital cavity and raised gently by a glass nerve dissector. Also, in 30 rats, $4 \mu \mathrm{L}$ cobra venom (mixture of $0.4 \mathrm{mg}$ lyophilized cobra venom; Venom Research Institute of Guangxi Medical University) was injected into the infraorbital nerve on the left side to establish a rat model of trigeminal neuralgia (10 rats) and two treatment groups, EA and pregabalin (10 rats in each group). For the sham-operated group (10 rats), rats underwent the same procedure except that saline was injected instead of cobra venom. The incision was closed using 5-0 absorbable sutures. Rats with obvious loss of vision in the eye on the lesion side were excluded.

\section{EA treatment}

For EA treatment, the acupuncture needle was quickly inserted into the left forelimb at the equivalent anatomic landmarks of human "Shousanli" (LI10) and "Quchi” (LI11) acupoints (Figure 1) after cleaning the rat's skin with alcohol swabs. ${ }^{17,18}$ The rats were put into a small plexiglass cage and they received EA stimulation using a Han's Acupoint Nerve Stimulator (HANS, LH series, Peking University). The frequency of EA stimulation was held to $2 / 100 \mathrm{~Hz}$ variable, which means 2 and $100 \mathrm{~Hz}$ shifting automatically; current intensity was maintained at $1 \mathrm{~mA}$ for 10 minutes, then increased to $1.5 \mathrm{~mA}$ for 10 minutes and finally increased to $2 \mathrm{~mA}$ for 10 minutes: the total procedure time was $30 \mathrm{~min}$ utes. If the needles were dropped during the procedure, they were inserted again as rapidly as possible. The EA treatment was given once every 2 days for 11 consecutive courses of treatment beginning on postoperative day 14 and ending on postoperative day 35 .

\section{Pregabalin treatment}

Pregabalin (75 mg; Pfizer, New York, NY, USA) was dissolved in $1 \mathrm{~mL}$ of $0.9 \%$ saline. The rats in the pregabalin group were treated with intragastric administration of pregabalin at a dose of $30 \mathrm{mg} / \mathrm{kg}$ by a self-made instrument once a day consecutively for 21 days following the 14 th day after operation. ${ }^{19}$ 

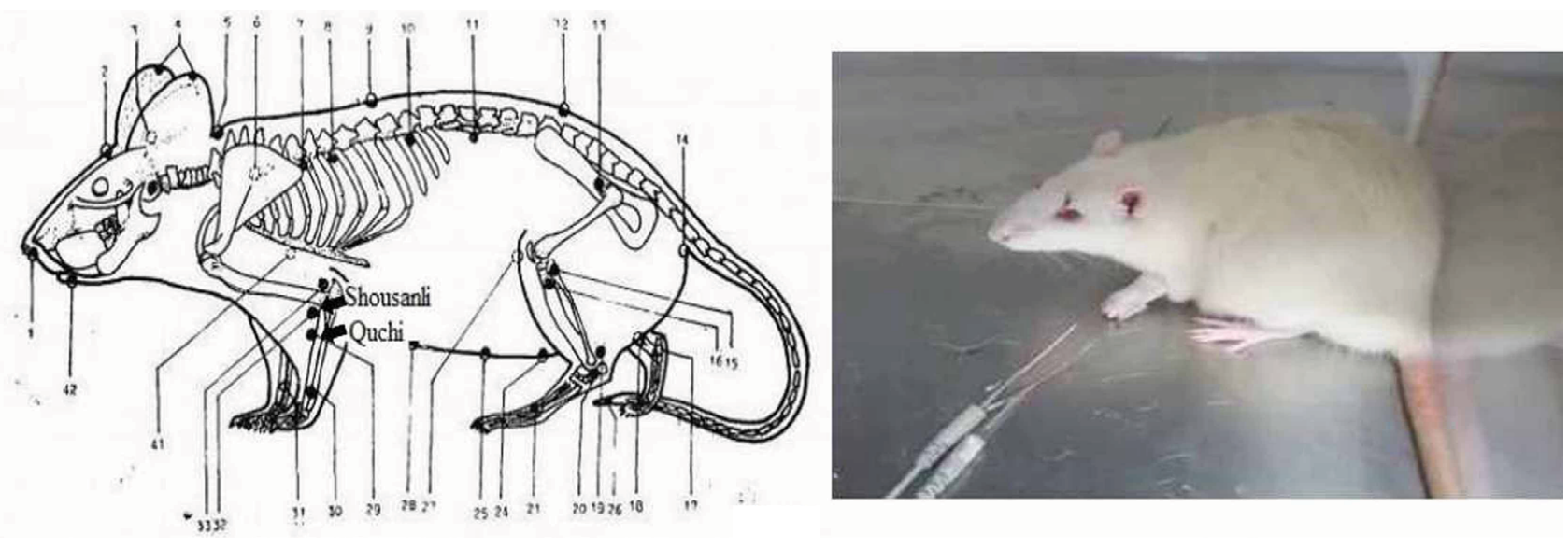

Figure I Pictures showing the EA points (I-42) and EA procedure.

Note: The rat showed no signs of distress during EA.

Abbreviation: EA, electro-acupuncture.

\section{Behavioral testing}

Neurobehavioral assessments were conducted prior to initiating treatment at preoperative day 3 and postoperative days 3 , $6,9,12,15,20,30$ and 40 . Rats were placed in a transparent plastic cage $(24 \times 35 \times 18 \mathrm{~cm})$ with a video camera placed $1 \mathrm{~m}$ in front of it. The rats' behavior was videotaped for a duration of 7 minutes. Behavioral changes such as frequency and length of exploratory and grooming behaviors of the rats were analyzed after video recording was completed. ${ }^{20}$

\section{The Morris water maze test}

The Morris water maze test was used to measure spatial learning and memory at 1 week before the operation and 1, 3 and 5 weeks after the operation, respectively. This test requires 4 days for the rats to learn and 1 day to test learning. It measures the efficiency of swimming to a hidden, submerged platform from four different locations in a circular tank filled with opaque and nontoxic water $\left(22^{\circ} \mathrm{C} \pm 1^{\circ} \mathrm{C}\right)$. Rats were placed into each of four different starting points for four training trials per day. In each trial, rats swam until they successfully located the hidden platform or were guided to it by the experimenter if not found within $60 \mathrm{~s}$. The experimenter kept the rats remaining on the platform for $30 \mathrm{~s}$ before removing them back to the cage, to ensure that they had access to spatial information during swimming and climbing onto the platform, prior to testing for spatial performance.

During the training trial, the length of the path and time taken by the rats to reach the platform was recorded by a computerized tracking system. Probe trials on day 5 were only $60 \mathrm{~s}$. The probe trial occurred $24 \mathrm{~h}$ after the final day of the training. The rats were started $180^{\circ}$ from the original platform position in the maze during the probe trial day while the hidden platform was removed and were then allowed to swim for $60 \mathrm{~s}$ in the tank. The average percentage time of the total probe trial time in the target quadrant and the number of exact site crossings were calculated. The average percentage of the total probe trial time in the target quadrant was calculated as a measure of spatial bias for the platform location.

\section{Electrophysiological recordings}

On postoperative day 50, electrophysiological recordings were made. Following halothane anesthesia, the brain was removed and the hippocampus was quickly dissected out with a Vibratome (VT 1000 S; Leica Microsystems, Wetzlar, Germany). Hippocampal slices (400 mm) were incubated in $20 \mathrm{~mL}$ artificial cerebrospinal fluid (ACSF; containing in mM: $125 \mathrm{NaCl}, 2.5 \mathrm{KCl}, 1.25 \mathrm{NaH}_{2} \mathrm{PO}_{4}, 25 \mathrm{NaHCO}_{3}, 10$ D-glucose, $2.5 \mathrm{CaCl}_{2}, 1.5 \mathrm{MgCl}_{2}, \mathrm{pH}=7$ ) saturated with $5 \%$ $\mathrm{CO}_{2}$ in $\mathrm{O}_{2}$ at a temperature $21^{\circ} \mathrm{C} \pm 1^{\circ} \mathrm{C}$ for at least $1 \mathrm{~h}$. Then, the prepared slices were transferred to a recording chamber, which was bubbled with oxygenated ACSF at a flow rate of 1.5-1.8 mL/minute with the $\mathrm{Ca}^{2+}: \mathrm{Mg}^{2+}$ ratio being 2.0:1.0 $\mathrm{mM}$ at a temperature of $31^{\circ} \mathrm{C} \pm 1^{\circ} \mathrm{C}$.

Recording pipettes were pulled from $110 \mathrm{~mm}$ long borosilicate glass capillaries (GB 150F-8P; Sutter instrument, Novato, CA, USA). The outside diameter of the glass tubes was $1.5 \mathrm{~mm}$. The pipettes were produced with electronic electrode extracting equipment (P97; Sutter instrument). The electrode resistance amounted to $3 \mathrm{M} \Omega$.

The field excitatory postsynaptic potential (fEPSP) was recorded with Axopatch 700B amplifier. Extracellular recording electrodes were filled with recording ACSF and placed in the stratum radiatum of CA1. The fEPSP responses were evoked by stimulation with a bipolar electrode placed on the CA3 area. Schaffer collateral pathway was tested during the 
experiments, and thus, stimulating electrodes were positioned 200-400 $\mu \mathrm{m}$ away from the recording electrodes.

Stimulation parameters consisted of single negative voltage pulses of $2-5 \mathrm{~V}$ of $100 \mu$ s duration. The slices were stimulated with single test pulses every $30 \mathrm{~s}$ for at least 30 minutes, followed by Theta-burst stimulation (TBS) and 60 minutes of test stimulation. All TBS contained 12 bursts at $200 \mathrm{~ms}$ interstimulation intervals and was repeated two times with $10 \mathrm{~s}$ intervals; each burst consisted of four pulses of 100 $\mathrm{Hz}^{21}$ The intensity of high-frequency tetanic stimulation is a critical variable for the induction of long-term potentiation (LTP). The magnitude of LTP typically increases in parallel with tetanus intensity. The higher the number of afferents activated, the more the number of synapses that reach the threshold for potentiation. Data were digitized at $10 \mathrm{kHz}$ and analyzed with clampfit 10.5 software. The time course of changes in the fEPSP slopes was calculated in relation to the signals obtained prior to TBS (100\%), normalizing all signals to this baseline and then averaging across experiments. The degree of LTP is expressed as percentage of the baseline level. For comparing the long-term synaptic plasticity between groups, data points were created by averaging the 20 signals at 51-60 minutes post-TBS in relation to the average of 20 signals during the last 10 minutes prior to TBS (100\%) and then averaging across experiments.

\section{Transmission electron microscopy}

At postoperative day 50, all rats were anesthetized by an intraperitoneal injection of $4 \%$ chloral hydrate. Subsequently, $0.9 \%$ saline solution $\left(37^{\circ} \mathrm{C} ; 250 \mathrm{~mL}\right)$ was perfused through the heart, followed by $4 \%$ paraformaldehyde in 0.1 $\mathrm{M}$ phosphate buffer $(\mathrm{pH} 7.4)$ at $2^{\circ} \mathrm{C}-4^{\circ} \mathrm{C}(100 \mathrm{~mL})$. The left Gasserian ganglion, medulla oblongata and hippocampus tissues were dissected free and fixed in 3\% glutaraldehyde at $4^{\circ} \mathrm{C}$ for at least $2 \mathrm{~h}$, then washed in $0.1 \mathrm{M}$ phosphate buffer three times and finally post-fixed in $1 \%$ osmium tetroxide for $2 \mathrm{~h}$. These tissues were subjected to dehydration, xylene transparent clearing, wax infiltration, paraffin embedding and sectioning into ultrathin sections $(50-70 \mathrm{~nm})$ for transmission electron microscopy (TEM; H-9000NARIbaraki; Hitachi Ltd., Tokyo, Japan).

\section{Statistical analysis}

All statistical analyses were performed using SPSS version 18.0 (SPSS Inc., Chicago, IL, USA). Two-way repeated measures analysis of variance (ANOVA) was used for analyzing data from the Morris water maze test. Other statistical tests were performed using one-way ANOVA for comparisons.
Within-group differences across time were evaluated by ANOVA. The post hoc test was LSD $t$-test. $P<0.05$ was considered as statistically significant. All data are expressed as mean $\pm \mathrm{SD}$.

\section{Results \\ Behavioral changes of trigeminal neuralgia model}

As shown in Figure 1, there were no differences among all groups in exploratory frequency and length $(P>0.05$; Figure $2 \mathrm{~A}$, $\mathrm{B})$ or grooming frequency and length $(P>0.05$; Figure $2 \mathrm{C}, \mathrm{D})$, respectively, before the operation and at postoperative day 3. At postoperative day 6 , rats injected cobra venom demonstrated $22.9 \%$ reduction in exploratory frequency and $18.9 \%$ relative reduction in exploratory length, compared with the rats in sham group (frequency: ${ }^{*} P=0.011$, length: ${ }^{*} P=0.041$; Figure $2 \mathrm{~A}, \mathrm{~B}$ ); meanwhile, the grooming frequency increased by $71.4 \%$ and the grooming length by $55.5 \%$ (frequency: ${ }^{*} P=0.003$, length: $* P=0.028$; Figure $2 \mathrm{C}, \mathrm{D})$.

In the EA group, the exploratory frequency increased by $60.7 \%$ and the length by $57.6 \%$ over time compared to the cobra venom group (frequency: $+P=0.040$, length: $+P=0.040$; Figure 2A, B), while the grooming frequency represented $44.4 \%$ reduction and the length showed $52.5 \%$ reduction (frequency: $+P=0.011$, length: $+P=0.002$; Figure $2 \mathrm{C}, \mathrm{D}$ ) at postoperative day 20 .

In the pregabalin group, the exploratory frequency of rats increased by $64.3 \%$ and the exploratory length by $61.5 \%$ over time compared with the cobra venom group (frequency: $P=0.038$, length: $-P=0.037$; Figure $2 \mathrm{~A}, \mathrm{~B}$ ), while the grooming frequency showed $75.6 \%$ relative decrease and the length produced $60.9 \%$ relative reduction (frequency: $-P=0.001$, length: $-P=0.001$; Figure $2 \mathrm{C}, \mathrm{D})$ at postoperative day 20 .

\section{Changes in spatial learning}

As shown in Figure 3A, B, 7 days prior to and after the operation, there were no differences among all groups in escape latency, indicating that all rats could find the submerged platform and the cues in the surrounding environment. The escape latency became shorter with increased training trials over time in all groups. As shown in Figure 3C, D, at 3 and 5 weeks after the operation, there were differences in escape latency. The escape latency of cobra venom group was significantly longer at 3 and 5 weeks after the operation to reach the platform, compared to the sham-operated group (week 3: ${ }^{*} P=0.002$, week 5 : ${ }^{*} P=0.011$; Figure $3 \mathrm{C}$, D), suggesting that the cobra venom rats exhibited a slower learning rate. 
A

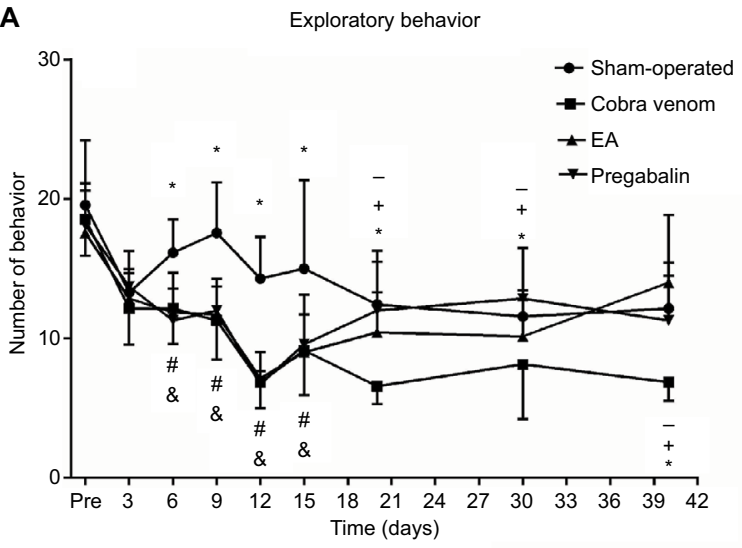

C

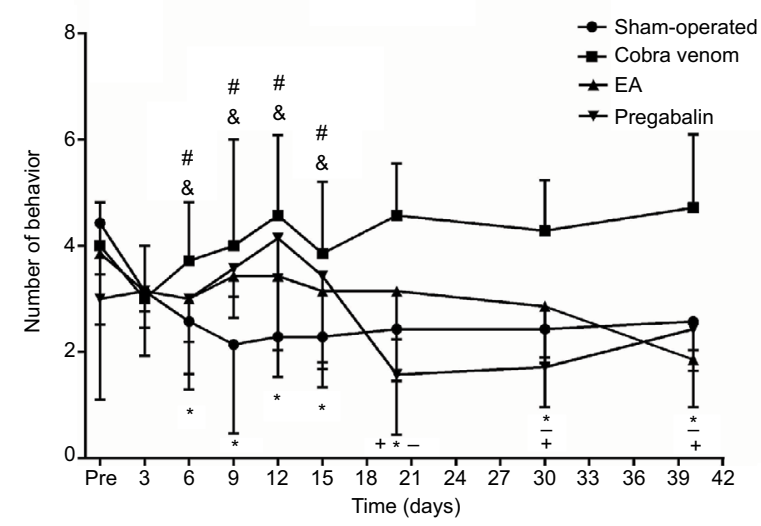

B

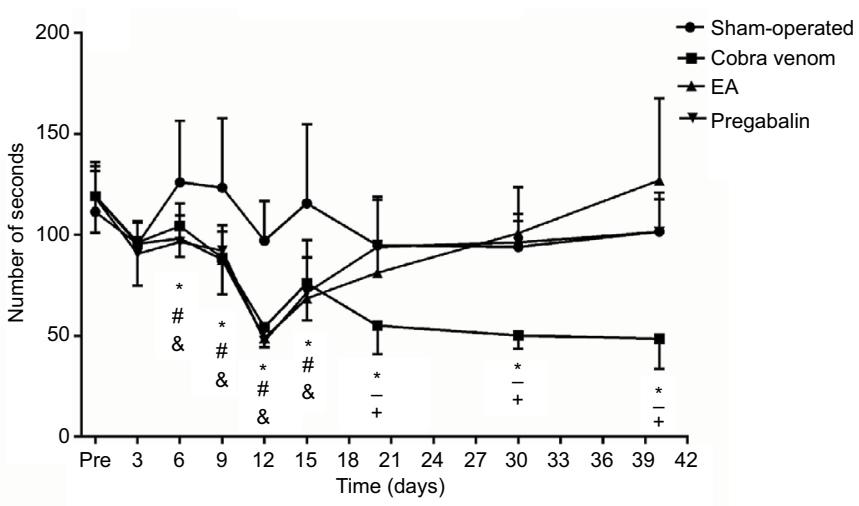

D

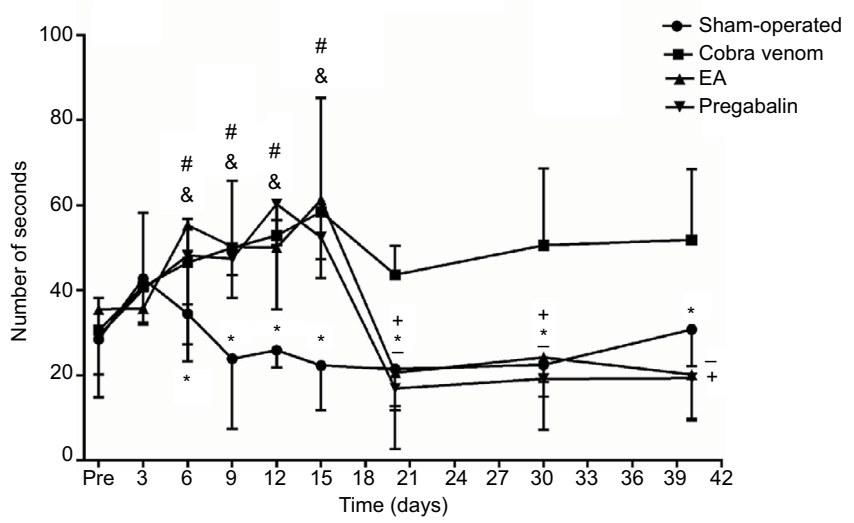

Figure 2 Frequency and length of face grooming and exploratory behavior were observed before the operation and at eight postoperative time points.

Notes: (A-D) No significant differences among groups were found before and 3 days after the operation $(P>0.05)$. At postoperative day 6 , the frequency and length of exploratory behavior of rats after cobra venom injection showed remarkable decrease in comparison to the rats in sham-operated group (*: compared between cobra venom and sham-operated groups, frequency: $* P=0.01 \mathrm{I}$, length: $* P=0.04 \mathrm{I} ;{ }^{*}$ : compared between $E A$ and sham-operated groups, frequency: ${ }^{\circledR} P=0.007$, length: ${ }^{*} P=0.024$; $\#$ : compared between pregabalin and sham-operated groups, frequency: ${ }^{*} P=0.002$, length: ${ }^{*} P=0.019$ ), while the frequency and length of grooming behavior showed increase in comparison to the rats in sham-operated group (*: compared between cobra venom and sham-operated groups, frequency: $* P=0.003$, length: * $P=0.028$; ${ }^{*}$ : compared between $E A$ and sham-operated groups, frequency: ${ }^{*} P=0.005$, length: ${ }^{*} P=0.002$; : compared between pregabalin and sham-operated groups, frequency: $P=0.01 \mathrm{I}$, length: ${ }^{\#} P=0.040$ ). Significant differences were observed at postoperative day 20 after EA and pregabalin treatment, respectively, compared to cobra venom group (*: compared between cobra venom and sham-operated groups, frequency of exploratory behavior: $* P=0.003$, length of exploratory behavior: $* P=0.012$, frequency of grooming behavior: $* P=0.03 \mathrm{I}$, length of grooming behavior: $* P=0.003 ;{ }^{+}$: compared between $E A$ and cobra venom groups, frequency of exploratory behavior: ${ }^{+} P=0.040$, length of exploratory behavior: ${ }^{+} P=0.040$, frequency of grooming behavior: ${ }^{+} P=0.01 \mathrm{I}$, length of grooming behavior: ${ }^{+} P=0.002 ;{ }^{-}$: compared between pregabalin and cobra venom groups, frequency of exploratory behavior: $P=0.038$, length of exploratory behavior: ${ }^{-P}=0.037$, frequency of grooming behavior: ${ }^{-P}=0.00 \mathrm{I}$, length of grooming behavior: ${ }^{-P}=0.00 \mathrm{I}$ ).

Abbreviation: EA, electro-acupuncture.

In the EA group, the escape latency dropped by $25.0 \%$ at 3 weeks after the operation and by $36.7 \%$ at 5 weeks after the operation compared with the cobra venom group (week 3 : $\& P=0.005$, week 5: $\& P=0.004$; Figure $3 \mathrm{C}, \mathrm{D})$ and there were no differences between the EA and sham-operated groups $(P>0.05$; Figure 3C, D). This suggests that EA-treated rats performed significantly better than cobra venom injected rats over time.

In the pregabalin group, the escape latency increased by $25.3 \%$ at 3 weeks after the operation and by $37.5 \%$ at 5 weeks after the operation compared to the sham-operated group (week 3: $\# P=0.048$, week 5: $\# P=0.033$; Figure 3C, $D)$, with no difference in spatial learning or memory when compared to the cobra venom group $(P>0.05$; Figure $3 \mathrm{C}, \mathrm{D})$.

\section{Changes in spatial memory through probe trials}

A probe trial test was conducted on the last day (day 5) at $24 \mathrm{~h}$ after the training. As shown in Figure 3E, F, at 7 days before and after the operation, all the groups showed no differences in the number of platform crossing and the average percentages of the total probe trial time in the target quadrant $(P>0.05$; Figure 3E, F). At postoperative days 21 and 35, the frequency of platform site crossings decreased by $58.8 \%$ at postoperative day 21 and by $48.3 \%$ on day 35 in the cobra venom group, compared with the sham-operated group (frequency of platform site crossings at postoperative day 21 : ${ }^{*} P=0.006$, at day $35: * P=0.021$; Figure $\left.3 \mathrm{E}\right)$. Meanwhile, the average percentages of the total probe trial time in the target 
A

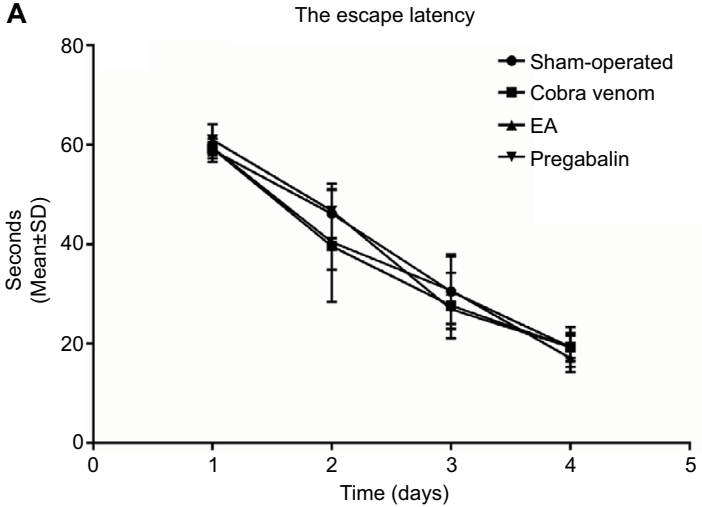

C

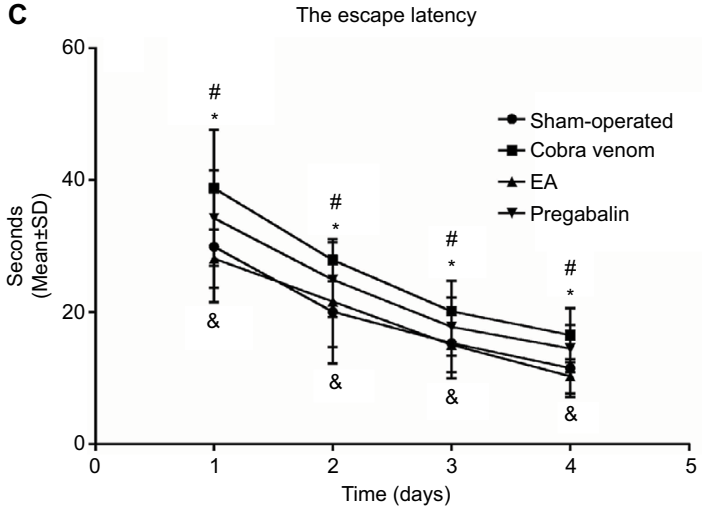

E

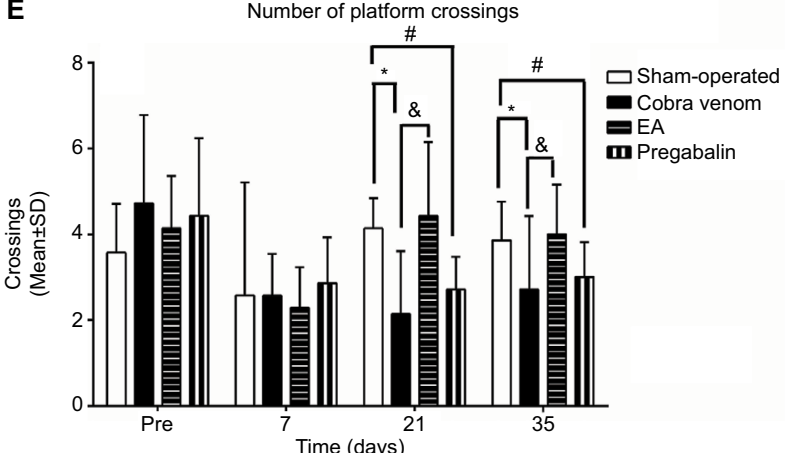

B

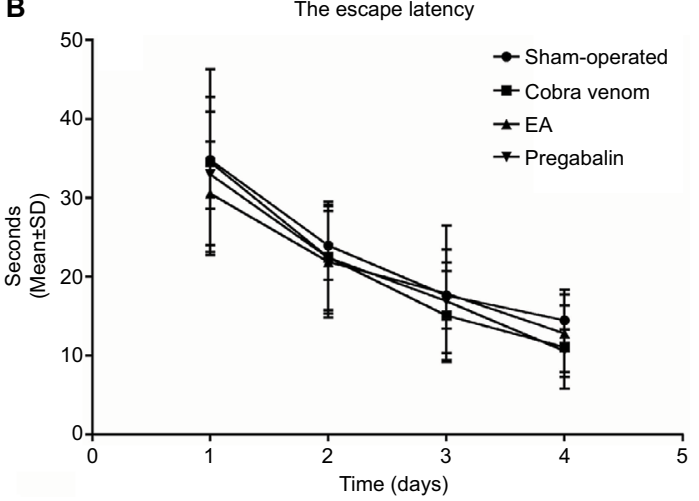

D

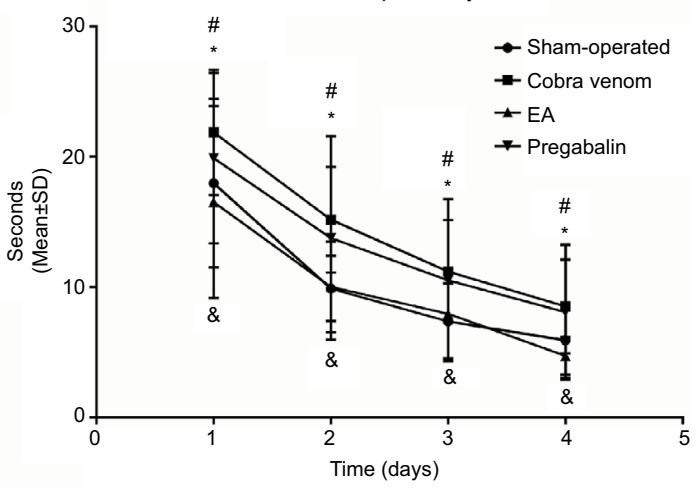

F

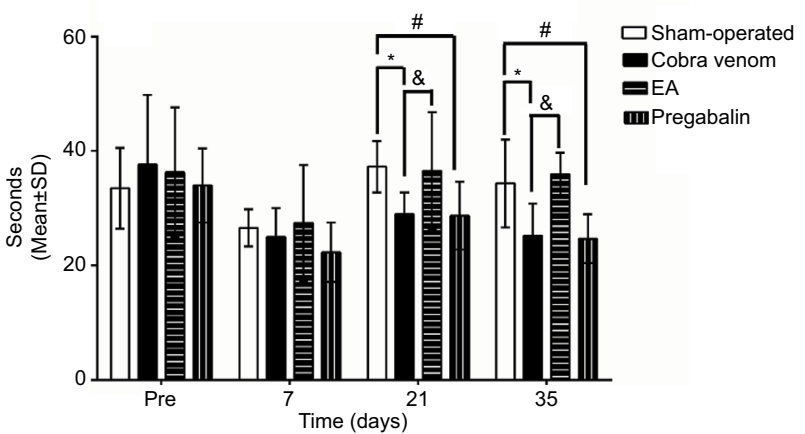

Figure 3 (A-D) Morris water maze training. (E, F) Morris water maze probe trial.

Notes: (A, B) No differences were found in the latency curve among all groups before the model was established and at I week after the operation. (C, D) At 3 and 5 weeks after the operation, the latency curve in the cobra venom and pregabalin groups showed a slower learning rate, compared to the sham-operated group (*: compared between cobra venom and sham-operated groups, ${ }^{*} P<0.05$; $:$ : compared between pregabalin and sham-operated groups, $\left.P<0.05\right)$. However, the EA group showed a faster learning rate compared with cobra venom group ( ${ }^{\star}$ : compared between EA and cobra venom groups, ${ }^{\&} P<0.05$ ). Data represent mean \pm SD (repeated measures one-way ANOVA followed by LSD $t$-test). (E) No differences were found in the number of platform crossings among all groups 7 days before and after the operation. At postoperative days $2 \mathrm{I}$ and 35, the number of platform site crossings showed a decrease in cobra venom group compared with that in the sham-operated group (*: compared between cobra venom and sham-operated groups, $* P<0.05$ ). However, the number of platform site crossings showed an increase in EA group compared with the cobra venom group $\left({ }^{\star}\right.$ : compared between EA and cobra venom groups, $\left.{ }^{\&} P<0.05\right)$. Meanwhile, the number of platform site crossings showed a decrease in pregabalin group compared with the shamoperated group (": compared between pregabalin and sham-operated groups, ${ }^{*}<0.05$ ), while there was no difference between EA and sham-operated groups or between cobra venom and pregabalin groups. (F) The average percentages of the total probe trial time in the target quadrant (without the platform). No differences were found in the average percentages of the total probe trial time in the target quadrant among all groups 7 days before and after the operation. At postoperative days 21 and 35 , the average percentages of the total probe trial time in the target quadrant showed a decrease in cobra venom group compared with that in sham-operated group (*: compared between cobra venom and sham-operated groups, $* P<0.05)$. However, the average percentages of the total probe trial time in the target quadrant showed an increase in $E A$ group compared with cobra venom group ( ${ }^{\&}$ : compared between EA and cobra venom groups, $\left.{ }^{\&} P<0.05\right)$. Meanwhile, the average percentages of the total probe trial time in the target quadrant showed a decrease in pregabalin group compared with sham-operated group ( ${ }^{*}$ : compared between pregabalin and sham-operated groups, $\left.{ }^{\#}<<0.05\right)$, while there was no difference between EA group and sham-operated group or between cobra venom group and pregabalin group.

Abbreviations: ANOVA, analysis of variance; EA, electro-acupuncture.

quadrant also decreased by $20.2 \%$ on postoperative day 21 and by $27.7 \%$ on day 35 in the cobra venom group, when compared to the sham-operated group (the average percentages of the total probe trial time in the target quadrant at postoperative day $21: * P=0.043$, at day $35: * P=0.002$; Figure $3 \mathrm{~F}$ ).
In the EA group, the number of platform site crossings increased by $106.7 \%$ at postoperative day 21 and by $46.7 \%$ at day 35 , compared to the cobra venom group (frequency of platform site crossings at postoperative day $21: \& P=0.002$, at day $35: \& P=0.012$; Figure $3 \mathrm{E}$ ), and the average percent- 
ages of the total probe trial time in the target quadrant rose by $20.9 \%$ on postoperative day 21 and by $29.2 \%$ on day 35 , compared to the cobra venom group (the average percentages of the total probe trial time in the target quadrant at postoperative day $21: \& P=0.036$, at day $35: \& P=0.001$; Figure $3 \mathrm{~F}$ ), but showed no significant difference when compared to the sham-operated group $(P>0.05$; Figure $3 \mathrm{E}, \mathrm{F})$.

However, in the pregabalin group, the number of platform site crossings decreased by $33.3 \%$ on postoperative day 21 and by $34.5 \%$ on day 35 , compared with the sham-operated group (frequency of platform site crossings at postoperative day 21: $\# P=0.041$, at day 35: $\# P=0.036$; Figure $3 \mathrm{E}$ ); also, the average percentages of the total probe trial time in the target quadrant dropped by $26.1 \%$ at postoperative day 21 and by $42.7 \%$ at day 35 , when compared to the sham-operated group (the average percentages of the total probe trial time in the target quadrant on postoperative day 21 : $\# P=0.044$, on day 35: $\# P=0.001$; Figure $3 \mathrm{~F}$ ), but showed no significant difference with the cobra venom group $(P>0.05$; Figure $3 \mathrm{E}, \mathrm{F})$.

\section{The ultrastructural changes}

As shown in Figure 4A, B, the myelin sheaths of the Gasserian ganglion or medulla oblongata were essentially of the same thickness and the structure of layers was clear and dense in the sham-operated group. In the cobra venom group, microscopic examination of Gasserian ganglion and medulla oblongata tissue showed myelinated fibers that were swollen and appeared in decreased density. This appeared as loose onion-like demyelination in the myelin sheath (Figure 4D, E). The ultrastructure of neurons in hippocampus was clearly altered. As shown in Figure 4C, the structure of cells was clear. As shown in Figure 4F, swelling
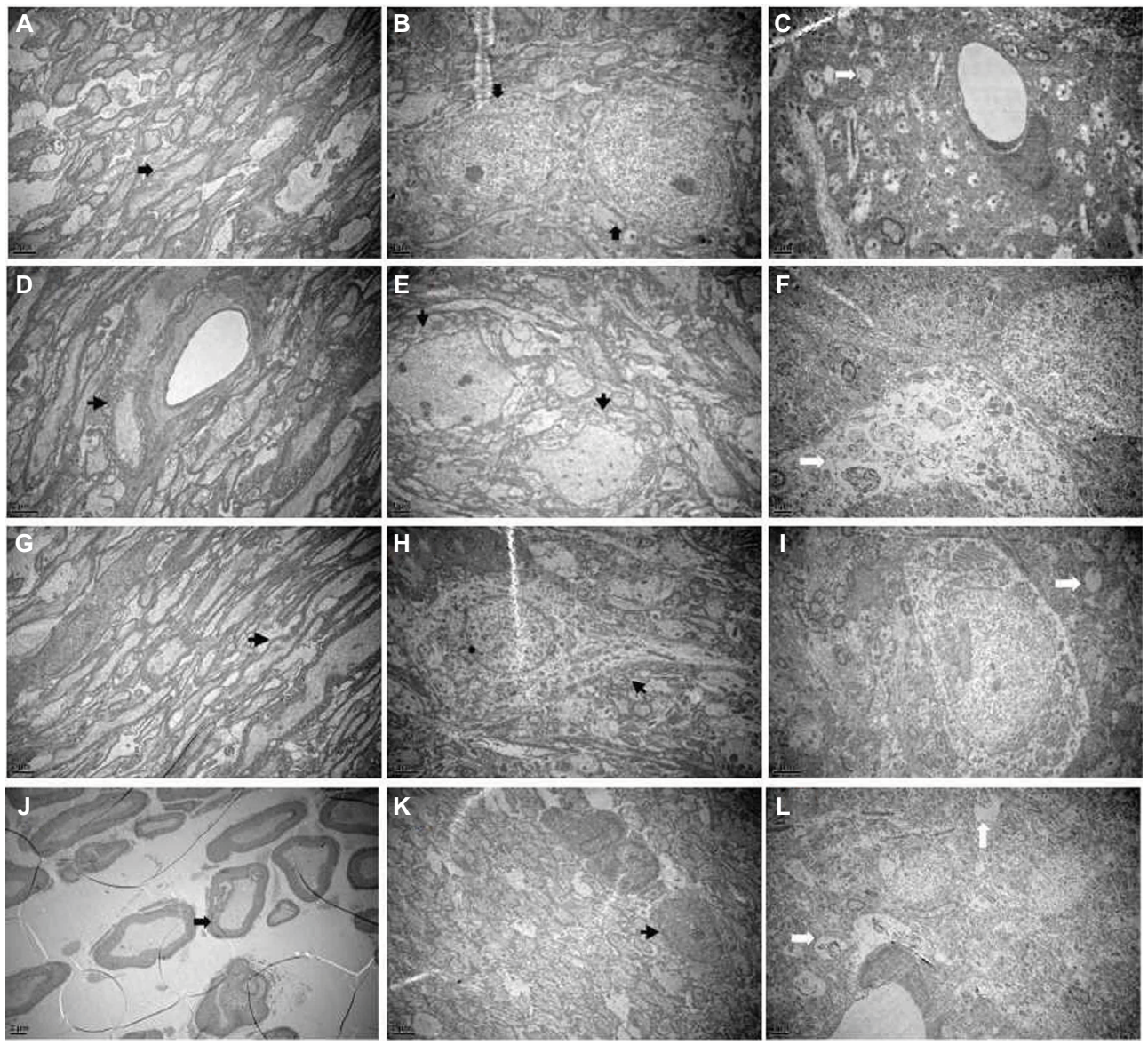

Figure 4 (A) The normal Gasserian ganglion, (B) medulla oblongata and (C) hippocampus. Electron microscopy showed that the structure of myelin sheaths and axon was entire (marked by arrows). (D) Gasserian ganglion, (E) medulla oblongata and (F) hippocampus with cobra venom injection. Electron microscopy showed severe demyelination and loss of axons (marked by arrows). The structure of myelin was disturbed and the layers were altered. The ultrastructure of neurons in hippocampus was altered obviously. Swelling of mitochondria and severe vacuolar degeneration in cells were observed. (G) Gasserian ganglion, (H) medulla oblongata and (I) hippocampus after EA treatment. Electron microscopy showed mild demyelination and loss of axons (marked by arrows). Microscopic examination of hippocampus neurons showed mild vacuolar degeneration and swelling of mitochondria in cells. (J) Gasserian ganglion, (K) medulla oblongata and (L) hippocampus after pregabalin treatment. Electron microscopy showed mild demyelination and loss of axons in part (marked by arrows). Microscopic examination of hippocampus neuron showed moderately severe vacuolar degeneration and swelling of mitochondria in cells.

Abbreviation: EA, electro-acupuncture. 
of mitochondria and severe vacuolar degeneration of cells were observed. In the EA group, nerves in the Gasserian ganglion and medulla oblongata showed mild onion-like demyelination, while the number of myelinating fibers was increased as compared to the cobra venom group (Figure $4 \mathrm{G}, \mathrm{H})$. Microscopic examination of hippocampal neurons showed mild vacuolar degeneration and swelling of mitochondria in cells (Figure 4I).

In the pregabalin group, the ultrastructure of nerves in the Gasserian ganglion and medulla oblongata showed mild onion-like demyelination, while the number of myelinating fibers demonstrated a mild loss that was less than that in the cobra venom group (Figure 4J, K). Microscopic examination of hippocampal neurons showed severe vacuolar degeneration and swelling of mitochondria (Figure 4L).

\section{The electrophysiological recordings}

As shown in Figure 5, the fEPSP slopes showing responses in rats in the sham-operated group were $128 \% \pm 4.32 \%$ in relation to the signals obtained prior to TBS (100\%), whereas the fEPSP slopes showing responses in rats in the cobra venom group significantly decreased to $110 \pm 5.57 \%(* P=0.029)$. In the EA group, the fEPSP slopes were $139 \% \pm 10.8 \%$ and showed a significant increase compared to the cobra venom group $(\& P=0.001)$, but showed no significant difference from the sham-operated group $(P>0.05)$.

In the pregabalin group, the fEPSP slopes were $109 \% \pm 4.01 \%$ and showed a significant decrease compared to the sham-operated group ( $\# P=0.046)$, but showed no significant difference from the cobra venom group $(P>0.05)$.

\section{Discussion}

EA and pregabalin are widely used clinically to treat trigeminal neuralgia. ${ }^{22,23}$ However, for understanding the analgesic and cognitive effects of EA and pregabalin on ameliorating chronic trigeminal neuralgia better, a simple and reliable animal model is required. Previously, our team established a trigeminal neuralgia model induced by cobra venom, which demonstrated reproducible changes in exploratory and grooming behavior. ${ }^{1,17}$ In this study, we applied our model to experimentally reproduce trigeminal neuralgia. On the third day after operation, the exploratory behavior of all cobra venom-injected rats showed a remarkable decrease in comparison to preoperative measures and the grooming behavior presented a significant increase. Results of this study were consistent with our previous findings. ${ }^{1,23}$ Furthermore, we
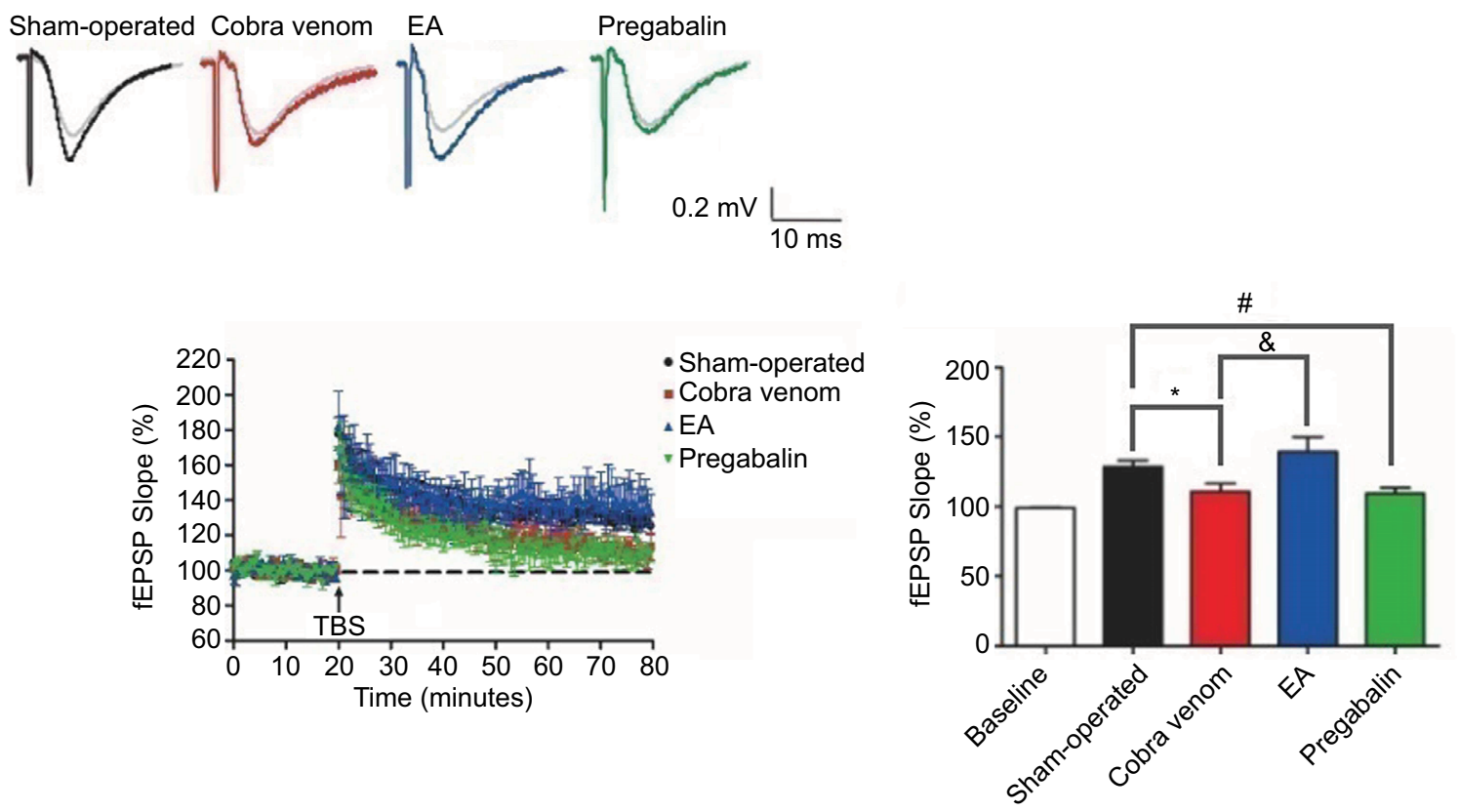

Figure 5 The fEPSP slope reveals differential time-dependent alternations in hippocampus CAI in different groups.

Notes: Vertical axis indicates the maximal fEPSP slope. Lateral axis indicates the time course of changes. Baseline is set at $100 \%$. Black curve, sham-operated slices ( $\mathrm{n}=10$ ); red curve, cobra venom slices $(n=10)$; blue curve, EA-treated slices $(n=10)$ and green curve, slices treated by pregabalin $(n=10)$. Data shown are mean $\pm S D$. $P<0.05$, significantly different as indicated. The four groups presented significant differences (ANOVA, two-tailed) in fEPSP slope at postoperative day 50, when compared with the baseline records. The fEPSP slope collected from the sham-operated group showed a significant increase compared to those collected from rats in the cobra venom group at the indicated times $(* P=0.029)$. Nevertheless, the $\mathrm{fEPSP}$ slope showing responses in rats in the EA group showed an increase compared to the cobra venom group ( $\left.{ }^{\circledR} P=0.00 \mathrm{I}\right)$, but showed no difference with the sham-operated group. Meanwhile, the fEPSP slope showing responses in rats in the pregabalin group showed a significant decrease compared to the sham-operated group $(\# P=0.046)$, while it showed no difference with the cobra venom group.

Abbreviations: ANOVA, analysis of variance; EA, electro-acupuncture; fEPSP, field excitatory postsynaptic potential; TBS, Theta-burst stimulation. 
also found that the Gasserian ganglion showed demyelinating ultrastructural alterations. In view of the above results, we believe that we successfully demonstrated a reproducible rat model of trigeminal neuralgia.

\section{The analgesic effects of EA stimulation and pregabalin}

In this study, we found that the rats showed clear behavioral changes including decreased grooming behavior and increased exploratory behavior after EA stimulation and administration of pregabalin, and there were no statistical differences between the two groups. In previous studies, the behavioral changes following the intervention with EA and pregabalin were also reported to decrease the grooming behavior and increase the exploratory behavior. ${ }^{17,19,21}$ The results of this study are consistent with these previous findings, indicating similar analgesic effects of EA stimulation and pregabalin in treating trigeminal neuralgia. Moreover, we found that the severe demyelinating phenomenon of Gasserian ganglion and medulla oblongata neurons decreased after EA and pregabalin intervention. We further confirmed a definite analgesic behavioral effect secondary to EA stimulation and pregabalin in trigeminal neuralgia. This suggests that inhibition of nerve demyelination might be the major mechanism of EA and pregabalin in alleviating the chronic trigeminal neuralgia induced by cobra venom.

\section{Cognitive effects of EA and pregabalin in a trigeminal neuralgia rat model induced by cobra venom}

Cognitive dysfunction is a common finding in chronic pain. Wu et al revealed that chronic trigeminal neuralgia induced by cobra venom in rats impairs spatial learning and memory function in the Morris water maze test. ${ }^{1}$ In this study, we also observed that the escape latency was increased and the number of platform crossings and the average percentages of the total probe trial time in the target quadrant showed a significant decrease in rats treated with cobra venom. Moreover, the electrophysiological LTP experiment in the hippocampal area of the brain confirmed changes consistent with decreased cognitive function in rats. LTP is an activity-dependent, long-lasting increase in the efficacy of synaptic transmission, and it is widely considered one of the major cellular mechanisms that underlie learning and memory. ${ }^{24,25}$ In this study, the results of LTP showed a marked reduction of fEPSP slope, which was measured by linear regression between two fixed time points in the cobra venom rats, compared with the sham-operated group. When the excitatory neurotransmitter is released from a preganglionic terminal and it binds to receptors on the postsynaptic dendritic membrane, it depolarizes that segment of the membrane generating an fEPSP. If enough fEPSPs arrive at the soma at the same time, and the amplitudes of the converging fEPSPs are sufficient to reach the action potential threshold, an action potential is generated and transmission of the preganglionic signal is complete. Thus, an increased fEPSP slope indicated higher synaptic transmission efficiency, while a decreased fEPSP slope indicated lower synaptic transmission efficiency. Furthermore, we also found that the hippocampal neurons showed a severe vacuolar degeneration phenomenon under electron microscopy. Vacuolar degeneration may be associated with aggregated tau hyperphosphorylation which may cause Alzheimer's disease. ${ }^{26}$ These findings indicated that trigeminal neuralgia induced by cobra venom in rats could lead to deficits in areas of the brain associated with the abilities of spatial learning and memory.

Meanwhile, we also found that the number of platform site crossings and the average percentages of the total probe trial time in the target quadrant showed an increase after EA intervention, compared with the cobra venom group. However, there were no differences in escape latency and the number of platform site crossings or the average percentages of the total probe trial time in the target quadrant between the pregabalin and cobra venom groups. These findings suggest that EA stimulation may improve the cognitive dysfunction of rats associated with cobra venom-induced trigeminal neuropathic pain, whereas pregabalin does not.

The causes of this phenomenon may be the following. Firstly, the fEPSP slope in ipsilateral hippocampus neurons had an increase after EA intervention, but the pregabalin group showed no significant increase. The increased fEPSP slope in the EA group indicated that EA could enhance the release of synaptic vesicles or increase the expression of postsynaptic density 95 to improve the efficiency of the synaptic transmission. ${ }^{27}$ Secondly, we observed that the vacuolation degeneration of hippocampal neurons could be attenuated significantly after EA stimulation; however, pregabalin does not change the ultrastructural alterations of hippocampal neurons. These results suggest that improvement in the synaptic transmission efficiency and vacuolation degeneration in the hippocampal neurons may be related to the mechanism of EA in improving the cognitive dysfunction in this experimental model.

Reduction in chronic pain may well result in amelioration of pain-induced cognitive dysfunction. Although 
pregabalin demonstrated a dramatic behavioral analgesic effect on chronic trigeminal pain in this study, it did not suppress the development of deficits in the abilities of spatial learning and memory. Both peripheral and central mechanisms are critical in understanding and treating neuropathic pain.

\section{Limitations}

In our study, the selection of acupoints in EA treatment group may have been limited by technical difficulties. Also, our results are dependent on our own experimental model producing changes such as trigeminal neuralgia in a clinical setting. Nonetheless, the relationship between trigeminal neuralgia, cognitive dysfunction and treatment options requires further study.

\section{Conclusion}

In brief, these data indicate that EA stimulation not only decreased neuropathic pain behavior induced by cobra venom, but also improved deficits in spatial learning and memory abilities induced by chronic trigeminal neuralgia. However, pregabalin only ameliorated the trigeminal neuropathic pain behavior induced by cobra venom without affecting the cognitive dysfunction induced by this experimental model.

\section{Acknowledgment}

This study was supported by the Capital Foundation of Medical Developments (CFMD).

\section{Disclosure}

The authors report no conflicts of interest in this work.

\section{References}

1. Wu Z, Qian XY, An JX, et al. Trigeminal neuralgia induced by cobra venom in the rat leads to deficits in abilities of spatial learning and memory. Pain Physician. 2015;18(2):E207-E216.

2. Liu X, Li L, Tang F, Wu S, Hu Y. Memory impairment in chronic pain patients and the related neuropsychological mechanisms: a review. Acta Neuropsychiatr. 2014;26(4):195-201.

3. Baliki MN, Geha PY, Apkarian AV, Chialvo DR. Beyond feeling: chronic pain hurts the brain, disrupting the default-mode network dynamics. $J$ Neurosci. 2008;28(6):1398-1403.

4. Lehmann TR, Russell DW, Spratt KF, et al. Efficacy of electroacupuncture and TENS in the rehabilitation of chronic low back pain patients. Pain. 1986;26(3):277-290.

5. White A. The fibromyalgia syndrome. Electroacupuncture is a potentially valuable treatment. BMJ. 1995;310(6991):1406.

6. Lee SH, Lee BC. Electroacupuncture relieves pain in men with chronic prostatitis/chronic pelvic pain syndrome: three-arm randomized trial. Urology. 2009;73(5):1036-1041.

7. Shen X, Xie YY, Chen C, Wang XP. Effects of electroacupuncture on cognitive function in rats with Parkinson's disease. Int J Physiol Pathophysiol Pharmacol. 2015;7(3):145-151.
8. Sun SZ, Cui ZY. [Effect of different needle retaining times of electroacupuncture on trigeminal neuralgia]. Zhong guo Zhen Jiu. 2011;31(3):213-215. Chinese.

9. Yang JX, Zhang JP, Yu JC, Han JX. [Clinical research of primary trigeminal neuralgia treated with electroacupuncture at qi streets acupoints combined with spinal regulation therapy]. Zhong guo Zhen Jiu. 2014;34(8):763-768. Chinese.

10. Lin R, Chen J, Li X, et al. Electroacupuncture at the Baihui acupoint alleviates cognitive impairment and exerts neuroprotective effects by modulating the expression and processing of brain-derived neurotrophic factor in APP/PS1 transgenic mice. Mol Med Rep. 2016;13(2):1611-1617.

11. Obermann M, Yoon MS, Sensen K, Maschke M, Diener HC, Katsarava Z. Efficacy of pregabalin in the treatment of trigeminal neuralgia. Cephalalgia. 2008;28(2):174-181.

12. Zakrzewska JM. Medical management of trigeminal neuropathic pains. Expert Opin Pharmacother. 2010;11(8):1239-1254.

13. Al-Quliti KW. Update on neuropathic pain treatment for trigeminal neuralgia. The pharmacological and surgical options. Neurosciences (Riyadh). 2015;20(2):107-114.

14. Liliana MT, Lacramioara O, Catalina Elena L, Andra Sabina NV, Gabriela R, Gratiela P. The effects of pregabalin on psycho-motor abilities and cognitive processes in mice. Rev Med Chir Soc Med Nat Iasi. 2015;119(1):185-192.

15. Salinsky M, Storzbach D, Munoz S. Cognitive effects of pregabalin in healthy volunteers: a double-blind, placebo-controlled trial. Neurology. 2010;74(9):755-761.

16. An JX, HeY, Qian XY, et al. A new animal model of trigeminal neuralgia produced by administration of cobra venom to the infraorbital nerve in the rat. Anesth Analg. 2011;113(3):652-656.

17. Liu H, Qian XY, An JX, et al. Analgesic effects and neuropathology changes of electroacupuncture on curing a rat model of brachial plexus neuralgia induced by cobra venom. Pain Physician. 2016;19(3):E435-E447.

18. Lao L, Zhang RX, Zhang G, Wang X, Berman BM, Ren K. A parametric study of electroacupuncture on persistent hyperalgesia and Fos protein expression in rats. Brain Res. 2004;1020(1-2):18-29.

19. Liu C, Qian X, JianXiong AN, et al. A new animal model of brachial plexus neuralgia produced by injection of cobra venom into the lower trunk in the rat. Pain Med. 2015;16(9):1680-1689.

20. Vos BP, Strassman AM, Maciewicz RJ. Behavioral evidence of trigeminal neuropathic pain following chronic constriction injury to the rat's infraorbital nerve. J Neurosci. 1994;14(5 Pt 1):2708-2723.

21. Zhao XY, Liu MG, Yuan DL, et al. Nociception-induced spatial and temporal plasticity of synaptic connection and function in the hippocampal formation of rats: a multi-electrode array recording. Mol Pain. 2009;5:55.

22. Luo Fei, An jian-xiong, Fan bi-fa, Han ji-sheng. Treatment of trigeminal neuraligia with transcutaneous electric acupoint stimulation on twentyeight patients. Chin J Pain Med. 1997;3:107-111.

23. Zhao QQ, Qian XY, An JX, et al. Rat model of trigeminal neuralgia using cobra venom mimics the electron microscopy, behavioral, and anticonvulsant drug responses seen in patients. Pain Physician. 2015;18(6):E1083-E1090.

24. Bliss TV, Collingridge GL. A synaptic model of memory: long-term potentiation in the hippocampus. Nature. 1993;361(6407):31-39.

25. Ohnami S, Kato A, Ogawa K, Shinohara S, Ono H, Tanabe M. Effects of milnacipran, a 5-HT and noradrenaline reuptake inhibitor, on C-fibreevoked field potentials in spinal long-term potentiation and neuropathic pain. Br J Pharmacol. 2012;167(3):537-547.

26. Hunter S, Minett T, Polvikoski T, Mukaetova-Ladinska E, Brayne C; Cambridge City over-75s Cohort Collaboration. Re-examining tauimmunoreactive pathology in the population: granulovacuolar degeneration and neurofibrillary tangles. Alzheimers Res Ther. 2015;7(1):57.

27. Zhang XJ, Wu Q. [Effects of electroacupuncture at different acupoints on learning and memory ability and PSD-95 protein expression on hippocampus CA1 in rats with autism]. Zhongguo Zhen Jiu. 2013;33(7):627-631. Chinese. 
The Journal of Pain Research is an international, peer reviewed, open access, online journal that welcomes laboratory and clinical findings in the fields of pain research and the prevention and management of pain. Original research, reviews, symposium reports, hypothesis formation and commentaries are all considered for publication
The manuscript management system is completely online and includes a very quick and fair peer-review system, which is all easy to use. Visit http://www.dovepress.com/testimonials.php to read real quotes from published authors. 